\title{
New And/Or Interesting in Microscopy at PITTCON 2001
}

As is our custom, and for the hopeful interest of our readers who could not attend the recent PITTCON 2001 Conference in New Orleans, we have attempted as follows to summary what was new and/or interesting in microscopy.

- Applied Scientific Instrumentation presented the FluarQuant package, a simple and easy to use photometry package built under LabView. FluarQuant allows the user to set up complex real-time experiments where fluorescently labeled cells can be excited at any wavelength and the quantity/ intensity of the emitted photons measured via a sensitive Photomultiplier Tube (PMT). FluarQuant can be integrated with one of ASl's automated $X Y$ stages, high-speed scanning monochromators, and $u$-Inspector microscope to provide a fully automated screening System.

ASl's new MS2000 XYZ stage provides sub-micron accuracy for the $x_{1} y$, and $z$ position of the microscope stage. All axes derive their precise control through the use of closed-loop DC servomotors employing high-resolution rotary encoders for positioning feedback. The $X Y$ stage utilizes crossed-roller slides, precision lead-screws, and zero-backlash miniature geared DC servomotors for smooth and accurate motion. The Z-axis motion is accomplished with ASl's proven line of closedloop drives, each custom fitted to the microscope. The MS2000 control accepts industry standard commands, provides automatic backlash correction, and allows RS-232 \& USB communication with a host computer. Optional linear encoders can be added to the unit to provide resolutions of $20 \mathrm{~nm}$.

Applied Scientific Instrumentation: (541)461-8181, www. ASlimaging.com

- Digital Instruments, Veeco Instruments Inc. held the fourth symposium on Atomic Force Microscopy (AFM). The Symposium, chaired by Monte Heaton of AFM leader Digital Instruments/Veeco Metrology Group, focused on a variety of applications of AFM $-3 / 4$ including biology, materials science, and polymers.

A special talk, given by Dr. Philip Russell of North Carolina State University, entitled "Scanning Probe/Atomic Force Microscopy and Scanning Electron Microscopy: Complementary Microscopy and Microanalysis" explained the advantages and complementary capabilities of AFM relative to other microscopies (SEM, TEM) and analytical techniques. This presentation is available for webcast viewing on www.webcasting.com/ pittcon through June 5, 2001 and is also available in VHS Video by contacting Digital Instruments, Veeco Metrology Group directly. Please email Mary McKeown-Christie (marym@di.com) with your request.

Veeco Instruments Inc.:(805)967-2700, www.veeco.com

- EDAX Inc., introduced its new products and technology including Genesis, a new software package which combines ease of use and full functionality enabling inexperienced and experienced users alike to obtain their required data, easily and rapidly. Genesis allows users to operate all EDAX software applications from a single screen. The user interface has been developed to allow the new or "casual" operators to be able to obtain materials characterization information easily whilst maintaining the full capabilities, recognized in our present EDS software, via easy to follow pull down menus and right button functionality. The Polaris ${ }^{\text {TM }} \mathrm{X}$-ray microcalorimeter, being jointly developed by EDAX Inc. and CSP, Germany is a supercon- ducting cryogen-free EDS Detector has produced spectra close to the goal of $15 \mathrm{eV}$ at $1.5 \mathrm{keV}$. The $\operatorname{maXXi}$ is the premier coating thickness measurement system for anyone providing plating services to the automotive, tools, and large electronic parts industry. This unit utilizes state-of-art Xenon gas proportional detector in conjunction with a small but powerful $50 \mathrm{KV} \mathrm{X}$-ray tube, fundamental parameter software and various mathematical algorithms coating determination. The CT3000, PMI Analyzer, incorporates, the worlds first Pocket PC, providing the highest level of flexibility, and ease of use, known worldwide. This revolutionary analyzer identifies, classifies, and performs chemical characterization of well over 280 alloy/steel standards and families maintained in its onboard library.

EDAX.Inc.: (201)529-4880, www.edax.com

- McCone Microscopes \& Accessories displayed the new Linkam Optical DSC600 microscope cell which integrates nicely with the new Olympus BX2 polarized light microscope line. The Linkam Optical DSC600 is a novel low cost single cell system which overcomes the problems of baseline drift due to different heat flows into the sample and reference crucible. The single cell approach means the furnace is much smaller than conventional systems, therefore the response time is much faster. Because of the compact size of the DSC stage, it is easily mounted in other instruments such as FTIR, Laser Raman, and Confocal microscopes. Also on display was the Linkam CSS450 Optical Shearing microscope stage attached to the Olympus $B \times 2$ polarized light microscope. This system is ideal for applications which require the sample to be sheared and heated simultaneously while under microscopic observation. Some existing applications include polymer, surfactant, and liquid crystal studies. Both systems are computer driven using LinkSys, a Windows based software system. One additional item to note is the new Olympus CH40POL-2 polarized light microscope. The CH40POL-2 is a low cost polarized light microscope complete with a Bertrand lens, rotating stage, $1^{\text {st }}$ order red compensator, 10,20 , and $40 \mathrm{X}$ strainfree objectives, $10 \times$ dispersion staining objective, and a polarizing swing-out condenser lens.

McCrone Microscopes \& Accessories: (800)662-8122, www/ mccrone.com

- Nanonics Imaging Ltd. presented NSOM/AFM-100 Confo$\mathrm{cal}^{\mathrm{TM}}$ microscope, which provides a unique combination of integrated near-field, conventional far-field, confocal and atomic force microscopy. The NSOM/AFM-100 Confocal $^{\mathrm{TM}}$ fits on any optical microscope, including upright microscopes for which no on-line AFM options exist. The NSOM/AFM-100 ${ }^{\mathrm{TM}}$ can be transparently integrated with simultaneous Raman micro imaging systems, laser scanning confocal and multi photon microscopes and other lensbased imaging systems.

Applications include micro lens and waveguide profiling of light distribution and topography, imaging electro-optical devices such as semiconductor lasers, micro-electronic circuits produced with CMP technology and $<0.25$ design rules together with electrical characterization, super resolution florescence imaging of biological structures in physiological fluid, super resolution chemical sensing, etc. 
Nanonics also produces the singular 3D Flat Scanner stage that can be placed on any optical microscope and can provide 3D motions from the millimeter to nanometer scale, with improvements for 3D optical sectioning, non-linear microscopy and laser tweezers.

Nanonics Imaging, Ltd.: +972-2-678-9573 (Israel), www.nanonics. co.il

- Oxford Instruments Analytical displayed INCACrystal, an exciting new product that performs fast and simple Electron Backscatter Diffraction (EBSD) on their popular INCA microanalysis platform. INCACrystal revolutionizes EBSD by providing a fast and easy-to-use system in which orientation maps are acquired at a speed of 45000 pixels per hour-at least twice as fast as any competing system. A new high sensitivity CCD camera uses an electron beam current of just $1 \mathrm{nA}$ to produce high-resolution images, at that speed, without sample damage. Pattern acquisition time, beam position and pattern processing are all optimized to produce fast and clear orientation maps. Phase discrimination is used to differentiate different phases such as $\mathrm{Fe}$ FCC and BCC. In addition gain boundary analysis, texture analysis and other parameters can be easily combined through a unique new flexible "Area Analysis". INCACrystal comes with many benefits including on-line help and the Navigator system that leads less expert users in a clear and structured way through the wide portfolio of analytical options. Data can be displayed and expoerted in numerous forms or stored and recalled for later analysis. Reports can be customized in seconds and the fast analysis uniquely available means a huge productiivity boost. All INCA Crystal systems can be extended to provide combined INCA EDSMDS/EBSC systems.

Oxford Instruments Analytical: (978)369-9933, www.oxfordinstruments.com

- Princeton Gamma-Tech (PGT) unveiled the latest software version for the new SPIRIT Microanalysis System. SPIRIT uses the latest Image and Position Tagged Spectrometry (PTS) hardware to acquire full multi-signal data from an SEM sample during rapid, video rate scans. During active beam scanning at rates up to 30 frames/sec, all data is collected simultaneously: two SEM signals, total $X$-ray signal for all elements, and multiple external signals. Imagine full frame $X$-ray mapping that appears in seconds for all elements and grows continuously as you watch. No longer do you have to wait while the system slowly scans the area to produce a spectrum image file. SPIRIT data collection is driven by 64,000 by 64,000 scan control that allows dynamic controlled magnification and digital panning within the extensive scan area.

Of particular interest to attendees at the conference was the popular PRISM 50, the largest active area EDS detector in the industry. PRISM 50 gives $X$-ray counting rates five times that of conventional detectors. PRISM 50 is a dream come true for microanalysis users: the same quality data in one fifth the time! The PRISM lineup of detectors offers the customer the best resolution at $115 \mathrm{eV}$ to the largest detector area at $129 \mathrm{eV}$ resolution.

Princeton Gamma-Tech: (609)924-7310, www.PGT.com

- Soft Imaging Systems introduced the KeenView Camera, now cameras for all TEM applications. The KeenView Camera provides unsurpassed versatility through high frame rates (up to $22 \mathrm{fps}$ ) and high sensitivity (fiber-optically coupled in on-site position). Coupled with the analySIS software, the automatic acquisition and montage of multiple images can provide images with highest resolution yet large field of view. Soft Imaging
Systems now offers a web-based front-end application for the database. This allows publishing for databases on the Internet or intranet, with access to the images and other data through a standard web browser. The database administrator can define the databases, as well as available fields for search routines. Databases can be protected through passwords to provide secure access to the images and data.

Soft Imaging Systems: (303)234-9270, www.soft-imaging.com

- Thermo NORAN introduced Compass-Component Analysis Software for automated extraction and display of principal component data, and the Quest Digital X-ray Microanalysis System. Using technology developed by and licensed from Sandia National Laboratories, COMPASS automatically analyzes a data acquired from a sample using Spectral Imaging software on Thermo NORAN's VANTAGE Digital X-Ray Microanalysis System. With no user intervention, COMPASS identifies and displays all the unique compounds in the Spectral Imaging data set. The number of phases that can be identified and displayed is unlimited.

Acquiring a spectrum at every point in an electron image, Spectral Imaging provides virtually unlimited ability to produce $\mathrm{x}$-ray maps and linescans, as well as spectral analysis of any portion of the image even after the sample has been removed from the electron microscope. With COMPASS users can then automatically identify and display all the unique compounds in the initial data set simply by running the COMPASS software program.

Geared for the basic materials analysis market, Quest is a full-featured microanalysis system that provides high-throughput digital $x$-ray spectral, mapping, and linescan acquisitions, and crystal clear electron image capture. Quest features true 32-bit architecture in a rock-solid platform for qualitative and quantitative analysis, digital imaging, $x$-ray mapping, and linescans. The system also includes several features normally not found on ) 836-4346, www.noran.com

- Thermo Spectra-Tech, has engineered a new fullfeatured, entry-level FT-IR microscope-the Centaur $\mu$ available through Thermo Nicolet and Thermo Mattson. The Centaurus microscope offers simultaneous view and IR capabilities. Transmission and reflection modes are standard, with optional ATR microscopy. The microscope uses patented Targeting technology, which includes a masking aperture before the sample, resulting in high quality spectra. Video and Contact Alert are integral features of the Centaurus. The standard transmission and reflection modes offer manual focus, while the ATR option employs auto focus, a manual stage, and a motorized stage. The ATR option is available with $\mathrm{ZnSe}, \mathrm{Si}$, and $\mathrm{Ge}$ crystals. A Triton ${ }^{\mathrm{TM}} 10 \mathrm{X}$ condenser optimizes the transmission throughput of the Centaurus and is removable for large clearance. The microscope is compatible with multi-sampling FT-IR systems and can be left- or right-mounted.

Thermo Spectra-Tech also manufactures the reflection-only $10 \mathrm{X}$ objective designed exclusively for the Thermo Nicolet Continupm microscope. The objective is mounted on a fixed, singleplace nose piece and has a working distance of $9 \mathrm{~mm}$. The $10 \mathrm{X}$ optics provide $100 \mathrm{X}$ magnification, a numerical aperture of 0.71 , and a sampling aperture of $200 \mu \mathrm{m}$, resulting in a higher signalto-noise ratio and enabling microanalysis of larger samples than most objectives.

Thermo Spectra-Tech: www.thermospectra-tech.com 